\title{
Inappropriate Utilization of Antibiotics in COPD Exacerbations
}

\author{
Yelda Varol ${ }^{1}$ (D), Zuhal Karakurt² (D), Ali Kadri Çırak ${ }^{1}$ (D), Hülya Doğan Şahin ${ }^{1}$ (D), Cenk Kıraklı (D), \\ Berna Kömürcüoğlu ${ }^{1}$ \\ 'Department of Pulmonology, University of Health Sciences, Dr. Suat Seren Chest Diseases and Surgery Training and Research \\ Hospital, İzmir, Turkey \\ 2Department of Pulmonology, University of Health Sciences, Süreyyapasa Chest Diseases and Surgery Training and Research Hospital,
} istanbul, Turkey

Cite this article as: Varol Y, Karakurt Z, Çırak AK, et al. Inappropriate utilization of antibiotics in COPD exacerbations. Turk Thorac J 2020; 21(6): 397-403.

Abstract OBJECTIVE: Most exacerbations are mild to moderate, and antibiotic treatment for acute exacerbations of chronic obstructive pulmonary disease (AECOPD) is recommended for patients with severe exacerbations or severe underlying chronic obstructive pulmonary disease (COPD). Therefore, we aimed to investigate the patient factors that are associated with the prescription of antibiotics for inappropriate indication in AECOPD.

MATERIAL AND METHODS: This study was an observational cross-sectional study conducted in an outpatient clinic. The patients diagnosed with AECOPD and prescribed an antibiotic by a pulmonary physician were enrolled in the study. These prescriptions were documented by the pharmacist who asked the patient about the three cardinal symptoms. Appropriate and inappropriate prescription groups were defined by the types of exacerbations, as defined by the Global Initiative for Chronic Obstructive Lung Disease (GOLD) COPD report.

RESULTS: There were 138 patients, predominantly male $(83 \%)$, with a mean age of $64( \pm 9)$ years. A total of $64 \%$ of the prescriptions were appropriate; however, there were $50(36 \%)$ patients with inappropriate antibiotic prescription according to the mentioned criteria. When we compared the patient factors between the appropriate and inappropriate antibiotic prescriptions, there was no statistically significant difference in terms of age, Forced expiratory volume in first second \% (FEV1\%) predicted, FEV1 ml, forced vital capacity (FVC) ml, FEV1/ FVC, and amount (packs/year) of smoking ( $p>0.05$ for all parameters). FVC\% was statistically significantly lower in the appropriate antibiotic prescription group compared with that in the inappropriate antibiotic prescription group $(\mathrm{p}=0.049)$.

CONCLUSION: This study shows that most pulmonary physicians have a tendency to prescribe antibiotics for AECOPD according to the defined GOLD criteria. However, some of the physicians also prefer to prescribe antibiotics self-directedly, irrespective of the GOLD criteria. A physician-based questionnaire can be completed for future studies to define the underlying reasons for antibiotic prescription demands for cases of mild AECOPD.

KEYWORDS: COPD, antibiotics, acute exacerbation, sputum, appropriate

Received: January 25, 2019

Accepted: December 16, 2019

\section{INTRODUCTION}

Chronic obstructive pulmonary disease (COPD) is the fourth leading cause of death worldwide [1]. The exacerbations associated with COPD are major drivers of morbidity, mortality, and cost [2]. Administration of antibiotics is considered in acute exacerbations of COPD (AECOPD), which represents a frequent clinical situation. However, antibiotic resistance is increasing in hospitals and communities. It has been revealed that antibiotic prescriptions without definite indications contribute significantly to this increase [3].

A retrospective cohort study identified that $85 \%$ of the 69,820 patients who were prescribed antibiotics were admitted for AECOPD in 360 hospitals [4]. This finding is surprising because there is limited data showing any evidence of the efficacy of antibiotic treatment for AECOPD. It is shown that about $70 \%$ of the exacerbations are infectious at the origin [5]. However, only $20 \%-30 \%$ of the exacerbations are associated with the detection of bacteria by the sputum culture [5]. Most AECOPDs are mild to moderate and the antibiotic treatment for AECOPD is recommended for patients with severe exacerbations or severe underlying COPD [6]. Several biomarkers of airway infection are being studied in AECOPD for appropriate antibiotic use. Studies of C-reactive protein have reported conflicting findings; procalcitonin, which may be of value in the decision to use antibiotics, is a marker that is more specific for bacterial infections [7-8]. The test for procalcitonin is expensive and not readily available in most outpatient settings [9].

The 2015 report of the Global initiative for Chronic Obstructive Lung Disease (GOLD) recommend using antibiotics for patients with the exacerbations of COPD who have three cardinal symptoms: increase in dyspnea, sputum volume, and

Address for Correspondence: Yelda Varol, University of Health Sciences, Dr. Suat Seren Chest Diseases and Surgery Training and Research Hospital, İzmir, Turkey

E-mail: yeldavatansever@hotmail.com

(0) Copyright 2020 by Turkish Thoracic Society - Available online at www.turkthoracj.org 
sputum purulence; presence of two of the cardinal symptoms if increased purulence of sputum is one of the two symptoms; or necessity of mechanical ventilation [10]. There are insufficient data supporting the over-prescription of antibiotics for AECOPD, and patient factors may be important in overprescribing. Therefore, we aimed to investigate the patient factors that are associated with the prescription of antibiotic for inappropriate indication in AECOPD. We hypothesized that the patient factors are associated with the prescription of antibiotics for inappropriate indications in patients with AECOPD in an outpatient clinic. The primary objective of our study was the prescription of antibiotics for inappropriate indication according to the GOLD criteria for AECOPD. The secondary objectives were to determine the patient factors that could lead to inappropriate antibiotic prescription.

\section{MATERIAL AND METHODS}

This was an observational cross-sectional study conducted in the outpatient clinic of the Faculty of Medical Sciences University of Health Sciences, Dr. Suat Seren Chest Disease and Surgery Training and Research Hospital between January 1, 2017 and November 1, 2018. Written informed consent for participation in the study was obtained from all the participants. The study was approved by the ethics committee of the Faculty of Medical Sciences University of Health Sciences, Dr. Suat Seren Chest Disease and Surgery Training and Research Hospital (Clinical trial no: NCT03077152). At all stages of the study, there was an adherence to the guidelines of the Declaration of Helsinki.

\section{Inclusion Criteria}

The patients were enrolled in the study when they were diagnosed with AECOPD according to the definition of the GOLD without respiratory failure or need for intermediate or intensive care and if they fulfilled the following criteria:

- Male or female patients with COPD, older than 40 years (according to GOLD criteria)

- Antibiotic prescription for AECOPD (ICD J44.1) in a chest diseases outpatient clinic

- Prescriptions written by pulmonologists

- Willingness to participate (pharmacists sought consent from patients who met the selection criteria)

\section{Exclusion Criteria}

Patients were excluded according to the following criteria:

\section{MAIN POINTS}

- $\quad$ This study showed that pulmonary physicians prescribed antibiotics with a $64 \%$ relevance with the GOLD criteria for the exacerbations of COPD.

- However, some of the physicians also prefer to prescribe antibiotics self-directedly, irrespective of the GOLD criteria.

- Future studies are needed to define the underlying reasons for antibiotic prescription demands for cases of mild AECOPD.

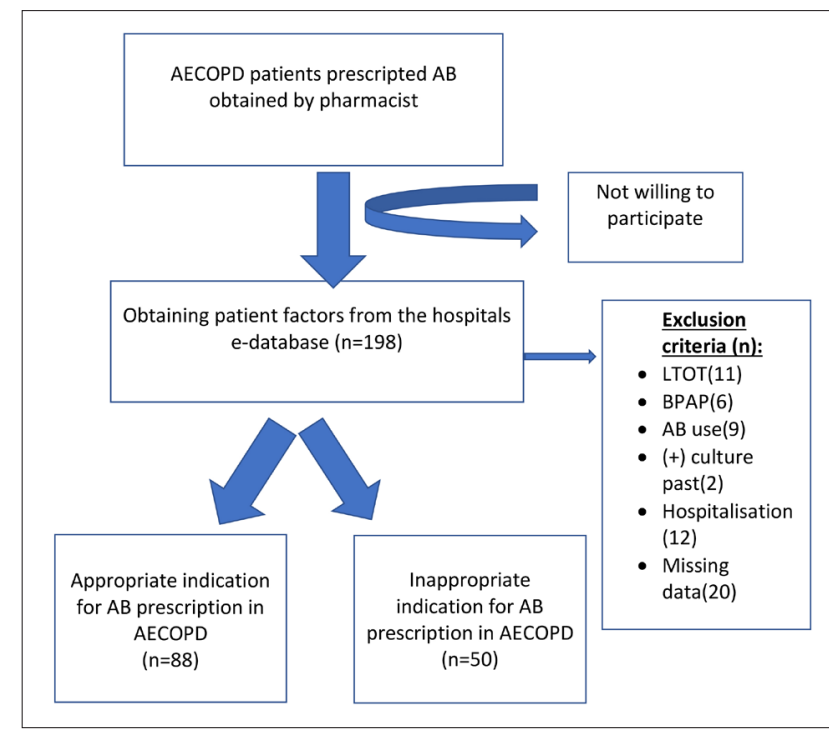

Figure 1. Flowchart of the study

- Patients using long-term oxygen treatment at home

- Patients using noninvasive mechanical ventilation for chronic hypercapnic failure at home

- Patients with a diagnosis of bronchiectasis, pneumonia, or lung cancer

- Those who had another need for antibiotic use (positive sputum culture in past 4 weeks or hospitalization for COPD in past 4 weeks)

- Missing data from the hospital's medical records (Figure 1).

\section{Subjects and Settings}

When patients with a diagnosis of COPD who have been prescribed an antibiotic by a pulmonologist go to the pharmacy, the pharmacist asks them the three cardinal symptoms of exacerbation according to GOLD report [10]. These cardinal symptoms are increase in dyspnea, sputum volume, and sputum purulence, and were recorded by the pharmacist. A manual for the protocol was written and used to inform the pharmacists. The pharmacists are trained to speak to the patient in a neutral manner to obtain written informed consent and also to keep patientsidentity document (ID) data secure. The pharmacists were trained to ask the questions within the time period planned, without affecting the time period of the patient to access his/her drug. Of the 10 pharmacists in the neighborhood area, five accepted to take part in this study. The conductor/ investigator of the study was provided the patient information, the ID, the written informed content, and a copy of the prescription from the pharmacy. The exclusion criteria were checked through the hospital's medical records. The age was calculated as DD/M/year and the smoking history was documented categorized as nonsmoker, ex-smoker, and current smoker. The comorbidities were saved using the Carlson comorbidity index score. The number of exacerbations in the past year, number of hospitalizations, and pulmonary function test results of the patients in the study were taken from the hospital database. The regular medication for COPD and coexisting diseases were obtained from the electronic database. The definition of appropriate and inappropriate use of antibiotics for AECOPD was defined by the criteria of GOLD COPD guidelines. According to these guidelines, antibiotics should be given to patients with exacerbations of COPD who have three 
Table 1. Demographic data of the study group

$\begin{array}{lc}\text { Nonsmoker, } \mathrm{n}(\%) & 2(1.4) \\ \text { Current smoker, } \mathrm{n}(\%) & 55(39.9) \\ \text { Ex-smoker, } \mathrm{n}(\%) & 81(58.7) \\ \text { Cigarette pack/year, mean (SD) } & 34( \pm 17) \\ \text { FEV1\% predicted } & 49( \pm 16) \\ \text { FEV1 mL } & 1372( \pm 500) \\ \text { FVC\% } & 61( \pm 16) \\ \text { FVC mL } & 2193( \pm 665) \\ \text { FEV1/FVC (\%) } & 59( \pm 9) \\ \text { Number of exacerbations in the past year } & 1( \pm 2) \\ \text { Number of hospitalizations in the past year } & 0( \pm 1) \\ \text { CIS: comorbidity index score; FEV1: forced expiratory volume in one } \\ \text { second; FVC: forced vital capacity }\end{array}$

Table 2. Prescription, regular medication, and exacerbation history of patients with AECOPD

\begin{tabular}{llcc} 
& & $\mathbf{n}$ & $\%$ \\
\hline Regular medication & LAMA & 13 & 9.4 \\
& LABA & 4 & 2.9 \\
& ICS+LABA & 16 & 11.6 \\
& Triple therapy & 63 & 45.7 \\
& Theophylline & 1 & 0.7 \\
& LABA+LAMA & 27 & 19.6 \\
& SABA+SAMA by nebulizer & 14 & 10.1 \\
Acute exacerbation & Severe & 46 & 33.3 \\
type & Moderate & 43 & 31.2 \\
& Mild & 49 & 35.5 \\
Antibiotic & Appropriate & 88 & 63.8 \\
prescription & Inappropriate & 50 & 36.2 \\
Antibiotic type & Penicillin & 12 & 8.7 \\
& Cephalosporin & 33 & 23.9 \\
& Quinolones & 44 & 31.9 \\
ICS: inhaled corticosteroids; LABA: long acting B2 agonists; LAMA:long & & \\
acting antimuscarinic & agent; SABA: short acting B2 agonists; SAMA: \\
short acting antimuscarinic agent; Triple therapy: LAMA+LABA+ICS & & \\
\hline Exacerbations $\geq 2$ & & 49 & 35.5 \\
& & 40 & 29.0
\end{tabular}

cardinal symptoms: increase in dyspnea, sputum volume, and sputum purulence; presence of two of the cardinal symptoms if increased purulence of sputum is one of the two symptoms; or necessity of mechanical ventilation (invasive or noninvasive) [10]. Therefore, appropriate use was if the prescription was written according to the GOLD report; in other words, if the patients had three of the cardinal symptoms or if they had two of the cardinal symptoms, of which one was increase in sputum purulence [10]. The information on the drug-drug interaction, defined as "a modification of the effect of a drug when administered with another drug" was obtained from the hospi- tal's electronic database [11]. The blood test results and chest $\mathrm{x}$-rays were viewed and analyzed for coexisting diagnosis of bronchiectasis, pneumonia, or lung cancer. Those patients with missing data were excluded from the study.

\section{Statistical Analysis}

The patients' demographics, pulmonary function test values, and the Charlson index scores are summarized in the descriptive analysis. The calculated sample size to estimate confidence interval $(\mathrm{Cl})$ of $15 \%$ around $25 \%$ prevalence of inappropriate prescription with $\mathrm{p}=0.05$ and power $80 \%$ was 128 . The study groups (inappropriate and appropriate prescription) were compared according to the parametric and nonparametric distribution of values. Normally distributed variables were compared with Student's t test and are shown as mean with standard deviation (SD). Non-normally distributed variables were compared by Mann-Whitney $U$ test and are shown as median interquartile ratio. For binary variables, the Chi-square test was used. We calculated the sample size to estimate the $\mathrm{Cl}$ of $15 \%$ around $25 \%-50 \%$ prevalence of inappropriate prescription with $\mathrm{p}=0.05$ and power $80 \%$. The data were analyzed using the Statistical Package for Social Sciences version 22.0 (IBM SPSS Corp.; Armonk, NY, USA).

\section{RESULTS}

During the study period, 186 eligible patients participated in the study; however, after exclusion, there were 138 patients with a mean age of $64( \pm 9)$. Of the 138 patients, $83 \%$ were male and $40 \%$ of the patients were current smokers with a mean of $34( \pm 17)$ packs/year. The demographic data of the study group is presented in Table 1 . The mean FEV1\% was 49 in the study population with a mean exacerbation history of $1( \pm 2)$ during the past year.

The number of patients with an inappropriate antibiotic prescription was $50(36 \%)$ according to the mentioned criteria. In $12(8.7 \%)$ patients, prescribing an antibiotic for AECOPD caused drug interaction. Most patients $(n=45 ; 7 \%)$ were taking triple therapy, consisting of long acting antimuscarinic agent (LAMA), long acting B2 agonists (LABA), and inhaler corticosteroids (ICS) as regular medication. About 35.5\% of the exacerbations were classified as mild. The most common antibiotics used for acute exacerbations were macrolides $(35.5 \%)$ and quinolones (31.9\%) (Table 2). In inappropriate antibiotic prescription group, $24 \%$ of the prescriptions were for quinolones, $46 \%$ for macrolides, $22 \%$ for cephalosporin, and $8 \%$ for penicillin.

When we compared the patient factors between the appropriate and inappropriate antibiotic prescriptions, there was no statistical significant difference in terms of age, FEV $1 \%$ predicted, FEV1 mL, FVC mL, FEV1/FVC, and amount (packs/ year) of smoking ( $p>0.05$ for all parameters) (Table 3 ). The FVC\% was statistically significantly lower in the appropriate antibiotic prescription group compared with that in the inappropriate antibiotic prescription group $(p=0.049)$. The gender and Charlson comorbidity index scores were not significantly different between the two groups (both, $p>0.05$ ). The history of exacerbations and hospitalization in the past year were also not different between the two groups (both, $\mathrm{p}>0.05$ ). The smoking status was not significantly different 
Table 3. Comparison of the patient factors between appropriate and inappropriate antibiotic prescription

\begin{tabular}{|c|c|c|c|c|c|}
\hline & \multicolumn{4}{|c|}{ Antibiotic prescription } & \multirow[b]{3}{*}{ p } \\
\hline & \multicolumn{2}{|c|}{ Appropriate } & \multicolumn{2}{|c|}{ Inappropriate } & \\
\hline & Mean & SD & Mean & SD & \\
\hline Age (years) & 63 & 9 & 65 & 9 & 0.20 \\
\hline FEV1 (\% predicted) & 47 & 17 & 51 & 16 & 0.12 \\
\hline FEV1 mL & 1335 & 510 & 1437 & 478 & 0.25 \\
\hline FVC (\% predicted) & 59 & 17 & 65 & 13 & 0.049 \\
\hline FVC mL & 2120 & 709 & 2320 & 564 & 0.09 \\
\hline FEV1/FVC (\%) & 60 & 9 & 59 & 9 & 0.70 \\
\hline Smoking (packs/year) & 34 & 14 & 35 & 21 & 0.67 \\
\hline
\end{tabular}

between the appropriate and inappropriate antibiotic prescription groups. The types of antibiotics were not different between the two groups (both, $\mathrm{p}>0.05$ ). The types of regular medication were not significantly different between the two groups $(p>0.05)$.

When we measured the association between the patient factors and outcome of the prescription of antibiotics for inappropriate indication, there was no statistically significant difference between the two groups in terms of age, FEV1\%, FEV1 $\mathrm{ml}, \mathrm{FVC} \mathrm{ml}, \mathrm{FEV} 1 / \mathrm{FVC}$, packs/year of smoking, and history of exacerbation. When we subgrouped the population into two according to the age $(\geq 65$ and $<65)$, there was no significant difference between the two groups in terms of inappropriate antibiotic prescription (both, $p>0.05$ ). The comparison of FEV $1 \%$ predicted subgroups ( $\leq 30,31 \%-50 \%$, $>50$ ) showed no difference in terms of inappropriate antibiotic prescription $(\mathrm{p}>0.05)$. In the study population, $82.6 \%$ of the patients had dyspnea, $62.3 \%$ had increase in the amount of sputum, and $46.4 \%$ had increase in the sputum purulence. There were 45 patients having all of these cardinal symptoms and 7 without having any of these symptoms. The Charlson comorbidity index score was higher in this group of patients $(n=7)$ having neither of the three cardinal symptoms compared with the ones with the symptoms; however, this difference was not significant $(p=0.39)$.

\section{DISCUSSION}

This study showed that pulmonary physicians prescribed antibiotics with a $64 \%$ relevance with the GOLD criteria for the exacerbations of COPD. In this study, the patient factors were not associated with the prescription of antibiotics for inappropriate indications in patients with AECOPD in the outpatient settings. Study groups with appropriate and inappropriate prescriptions were similar according to the age, gender, smoking status, pulmonary function test results, Charlson comorbidity index scores, and history of exacerbations.

Bont et al. [12] showed that Dutch general practitioners prescribe antibiotics for most elderly patients with acute bronchitis and in half of the cases with episodes of exacerbations of COPD. Bont et al. [12] indicated that only age was an independent determinant of antibiotic use in acute bronchitis (odds ratio $[\mathrm{OR}]=1.03 ; 95 \% \mathrm{Cl}, 1.003-1.048$ ), whereas in exacerbations of COPD, antibiotics were more often prescribed to male patients $(\mathrm{OR}=1.3 ; 95 \% \mathrm{Cl}, 1.0-1.5)$. The guidelines made for risk-stratified antibiotic treatment seems to be useful for purulent episodes of AECOPD [13]. However, these guidelines have not been prospectively validated for the elderly population [13]. In the present study, the study group consisted of relatively elderly and predominantly male COPD patients. We speculate that this age and gender dominance may influence the decision to prescribe antibiotics by the pulmonary physicians.

In a study by Montserrat-Capdevila, smoking (smoker/exsmoker) $(\mathrm{OR}=2.00)$ and forced vital capacity $(\mathrm{OR}=0.98)$ were found to be two of the factors associated with very frequent exacerbations $(>3)$ during the second year of follow up [14]. In the present study, $98 \%$ of the study population were current or ex-smokers. It can be argued that having a smoking history may have an effect on the physician's decision for prescribing antibiotics to patients with AECOPD.

In addition, Tupper et al. [15] studied the predictors of COPD in symptomatic smokers and ex-smokers who were seen in primary care and found that being a current smoker $(\mathrm{OR}=1.2 ; 95 \% \mathrm{Cl}, 1.01-1.5)$ and having self-reported dyspnea $(\mathrm{OR}=1.7 ; 95 \% \mathrm{Cl}, 1.4-2.0)$, wheeze $(\mathrm{OR}=1.9$; $95 \% \mathrm{Cl}, 1.5-2.3)$, and sputum $(\mathrm{OR}=1.4: 95 \% \mathrm{Cl}, 1.1-1.7)$ were associated with a significantly higher risk of being diagnosed with COPD. The country in which the present study was conducted has high value for smoking in general population. The COPD patients with smoking history, especially current smokers with COPD, may be more symptomatic during their usual days without needing to report it to their physicians in acute exacerbations. Therefore, physicians may have had a trend to write antibiotics to current smokers in the AECOPD patients.

According to the GOLD 2018 report, triple (ICS+LAMA+LABA) therapy is suggested to the COPD patients as a regular medication if the patients still have symptoms or if the patients continue to have exacerbations while they are under LABA+LAMA treatment [16]. In this study, most of the patients were using triple therapy as regular medication. Regarding the regular medications of the patients, we may predict that half of the 
patients with COPD were severe and symptomatic in this study population.

To help control the incidence of antibiotic resistance, limiting unnecessary use of antibiotics in the exacerbations of COPD is important. When the exacerbations of COPD are bacterial in origin, the antibiotic therapy seems appropriate. However, variety of exacerbations are caused by viral infections or even with no infection and have no need for antibiotic treatment. Furthermore, there is a growing incidence of resistance to common antibiotics [3]. A Cochrane Database metanalysis showed that in the exacerbations of COPD associated with increased cough and sputum purulence, the antibiotic therapy, regardless of choice, significantly decreases short term mortality, treatment failure, and sputum purulence [17]. However, this effect was greater in severe group of patients. In this metanalysis, only 2 of the 11 included studies were conducted in the community (Anthonisen 1987; Jørgensen 1992). In this metanalysis, the authors showed that when the analysis was restricted to community-based studies, there were no differences in the effects between the antibiotic and placebo [17]. In the present study, even the mild exacerbation group received an antibiotic for their exacerbation in an outpatient clinic.

There are only few studies that have compared the antibiotic treatment with the placebo in the AECOPD patients. Anthonisen et al. [18] showed a 55\% success rate with the placebo and a $68 \%$ response rate with the antibiotic treatment. Anthonisen et al. [18] also found that the patients who had increases in the amount of sputum, purulence of sputum, and dyspnea (Type 1 exacerbations) benefited the most ( $63 \%$ of the antibiotic treated exacerbations and $43 \%$ of the placebo treated exacerbations). The ones who met two of the three criteria (Type 2 exacerbations) showed an intermediate benefit, which was not statistically significant $(70 \%$ resolving on the antibiotics vs $60 \%$ resolving on the placebo). Those who met one of the above three criteria (Type 3 exacerbations) did not show any benefit (74\% resolving on the antibiotics vs $70 \%$ resolving on the placebo) [18]. Jorgensen et al. [19] compared the success rates of the placebo (63\%) and amoxicillin (64\%) in the treatment of uncomplicated exacerbations of chronic bronchitis. In 2010, using a standardized treatment including steroids, Daniels et al. [20] found clinical success in $61 \%$ of the patients in the doxycycline group and $53 \%$ in the placebo group. The limitations with these studies are the small number of participants and lack of optimization of the standard background care for AECOPD. Also, in the present study with outpatient settings, pulmonary physicians prescribed antibiotics with a $64 \%$ relevance according to the GOLD criteria for the exacerbations of COPD.

Salwan et al. [21] studied the prediction of prescribing antibiotics to AECOPD patients by general practitioners and found that the chest findings, increased CRP value, and decreased oxygen saturation were stronger predictors of prescribing the antibiotics and systemic corticosteroids than were the respiratory symptoms. In the present study, unless the patient factors did not have an influence on the antibiotic prescription, there could be other factors making the pulmonary physician prescribe an antibiotic for mild exacer- bations. It can be speculated that the physician could prescribe antibiotic for preventive purpose for severe AECOPD. The present study also shows that the two groups were quite similar in terms of the age, gender, comorbidity, COPD severity, and exacerbation history. This could be explained as the pulmonary physician might prescribe antibiotics to these kinds of patient groups without asking three cardinal symptoms in their outpatient clinic. Although the studies have conflicting results, we believe that the pulmonary physician may use biomarkers of airway infection (CRP or procalcitonin) for deciding to prescribe antibiotics for AECOPD, which were not included in preset study. In a recent study published by Butler et al., it was shown that CRP guided prescription of antibiotics for the exacerbation of COPD in primary care clinics resulted in a lower percentage of patients receiving the antibiotic prescription with no evidence of harm. New randomized controlled studies similar to the one mentioned earlier may guide the appropriate use of antibiotics in acute exacerbation of COPD [22].

In this study, the only statistically significant difference between the two groups was the predicted $\mathrm{FVC} \%$. FVC\% was significantly lower in the appropriate antibiotic prescription group compared with that in the inappropriate antibiotic prescription group. Montserrat-Capdevila et al. showed that FVC was a predictor of being a frequent exacerbator in the patients with COPD [14]. However, only one parameter of pulmonary function test may be statistically but not clinically relevant in the decision of prescribing antibiotics. These patients with low FVC may be more symptomatic, and therefore, test results can make the pulmonary physician to prescribe antibiotics.

Which antibiotic treatment should be selected to be prescribed for AECOPD is also a matter of debate [23]. A study showed that with the most potent broad-spectrum antibiotics, the patients are likely to get the best benefit from early treatment, such as amoxicillin/clavulanate and respiratory fluoroquinolones [24]. Philips et al. [25] studied either cefpodoxime proxetil $(200 \mathrm{mg}$, bid) or cefaclor $(250 \mathrm{mg}$, tid) regimen for 10 days in acute bacterial exacerbations of COPD and found that there were no statistically significant differences between the two drug regimens in the eradication of the initial pathogen (cefpodoxime, 91\%; cefaclor, 92\%) or end-of-therapy clinical response (cure and proved; cefpodoxime, 99\%; cefaclor, 92\%) rates for the evaluable patients. In the present study, the most common antibiotics used for acute exacerbations were macrolides, quinolones, cephalosporins, and penicillin. A study protocol was published by Rohde et al. [23] in 2015 questioning whether antibiotics are really needed in a well-defined patient cohort receiving state-of-the-art treatment in all other aspects in moderate exacerbation of COPD using oral sultamicillin vs placebo for 5 days. The results of this published protocol may help to give an answer for the efficacy of antibiotics in moderate exacerbation in patients with COPD.

Drug interactions, which can have unwanted effects, increases with the number of drugs taken [26]. In elderly patients, as the medication taken increased, the likelihood of the drug interactions increased [26]. In the present study, $8.7 \%$ of the 
patients had drug interaction because of prescribing antibiotics for AECOPD. However, the potential side effects of this interaction may not be clinically meaningful. In order to solve this problem, planning an electronic prescribing system can alert the physician early on to possible interactions [26].

In regard of this study, we had some limitations. Firstly, this study just determined an association, not causality. However, the study results can be important for showing the pulmonary physicians' demand of antibiotic prescription for mild AECOPD. Secondly, the Anthonisen criteria are subjective as the patients can have different mannerisms toward their doctors and pharmacists, and thus, we might not have been able to determine if the patient was truthful to the pharmacist. Furthermore, all the pharmacists involved in the present study were educated by the authors. Thirdly, nearly $30 \%$ patients were excluded from the study owing to missing data and all the patient files were obtained from the hospital electronic data base system to prevent entering erroneous data. However, the included number of the participants fulfilled the expected sample size. Finally, although the current guidelines for the treatment of COPD support using the three parameters created by Anthonisen, these criteria for identifying inappropriate antibiotic prescription for AECOPD are still debatable. This can be the reason why the physicians in the study prescribed antibiotics for patients with mild AECOPD in an outpatient clinic.

According to the findings of the present study, most pulmonary physicians have a tendency to prescribe antibiotics for AECOPD according to the defined Anthonisen criteria. However, some of these physicians also prefer to prescribe antibiotics self-directedly, irrespective of the Anthonisen criteria. A physician-based questionnaire can be completed for future studies to define the underlying reasons of antibiotic prescription demands in mild AECOPD.

Ethics Committee Approval: The study was approved by the ethics committee of the Faculty of Medical Sciences University of Health Sciences, Dr. Suat Seren Chest Disease and Surgery Training and Research Hospital (Clinical trial no: NCT03077152). At all stages of the study, there was an adherence to the guidelines of the Declaration of Helsinki.

Informed Consent: Written informed consent was obtained from all patients participating the study.

Peer-review: Externally peer-reviewed.

Author Contributions: Concept - Y.V.; Design - Y.V.; Supervision Z.K., C.K.,B.K.; Resources - Y.V.; Materials - H.D.S.; Data Collection and/or Processing - H.D.S., Y.V.; Analysis and/or Interpretation - Z.K., C.K.; Literature Search - A.K.C., B.K.; Writing Manuscript - Y. V., Z.K.; Critical Review - B.K.

Conflict of Interest: The authors have no conflicts of interest to declare.

Financial Disclosure: The authors declared that they have received no financial supportregarding this manuscript.

\section{REFERENCES}

1. Chronic Obstructive Pulmonary Disease Data Fact Sheet, NIH Publication 03-5229.
2. Mannino DM, Buist AS. Global burden of COPD: risk factors, prevalence, and future trends. Lancet 2007;370:765-73. [Crossref]

3. Chen DK, McGeer A, de Azavedo JC, et al. Decreased susceptibility of Streptococcus pneumoniae to fluoroquinolones in Canada. Canadian Bacterial Surveillance Network. N Engl J Med 1999;341:233-9. [Crossref]

4. Lindenauer PK, Pekow P, Gao S, et al. Quality of care for patients hospitalized for acute exacerbations of chronic obstructive pulmonary disease. Ann Intern Med 2006;144:894-3. [Crossref]

5. Celli BR, Barnes PJ. Exacerbations of chronic obstructive pulmonary disease. Eur Respir J 2007;29:1224-38. [Crossref]

6. Rohde GG. Prudent use of antibiotics: acute exacerbation of COPD as an example. Eur Respir J 2010;36:983-5. [Crossref]

7. Clark TW, Medina MJ, Batham S, et al. C-reactive protein level and microbial aetiology in patients hospitalised with acute exacerbation of COPD. Eur Respir J 2015;45:76-6. [Crossref]

8. Peng C, Tian C, Zhang Y, et al. C-reactive protein levels predict bacterial exacerbation in patients with chronic obstructive pulmonary disease. Am J Med Sci 2013;345:190-4. [Crossref]

9. Christ-Crain M, Jaccard-Stolz D, Bingisser R, et al. Effect of procalcitonin-guided treatment on antibiotic use and outcome in lower respiratory tract infections: cluster-randomised, singleblinded intervention trial. Lancet 2004;363:600-7. [Crossref]

10. Global strategy for the diagnosis, management, and prevention of chronic obstructive pulmonary disease. 2015 Report. Management of exacerbations. P:42-43.

11. Yıldırım AB, Kılınç AY. Polypharmacy and drug interactions in elderly patients. Turk Kardiyol Dern Ars 2017;45:17-21. [Crossref]

12. Bont J, Hak E, Birkhoff $\mathrm{CE}$, et al. Is co-morbidity taken into account in the antibiotic management of elderly patients with acute bronchitis and COPD exacerbations? Fam Pract 2007;24:317-22. [Crossref]

13. Albertson TE, Louie S, Chan AL. The diagnosis and treatment of elderly patients with acute exacerbation of chronic obstructive pulmonary disease and chronic bronchitis. J Am Geriatr Soc 2010;58:570-9. [Crossref]

14. Montserrat-Capdevila J, Godoy P, Marsal JR, et al. Risk factors for exacerbation in chronic obstructive pulmonary disease: a prospective study. Int J Tuberc Lung Dis 2016;20:389-95. [Crossref]

15. Tupper OD, Kjeldgaard P, Løkke A, et al. Predictors of COPD in symptomatic smokers and ex-smokers seen in primary care. Chron Respir Dis 2018;15:393-99. [Crossref]

16. Global strategy for the diagnosis, management, and prevention of chronic obstructive pulmonary disease. 2018 Report. Pharmacologic therapy for stable COPD. P:53

17. Ram FS, Rodriguez-Roisin R, Granados-Navarrete A, et al. Antibiotics for exacerbations of chronic obstructive pulmonary disease. Cochrane Database Syst Rev doi:10.1002/14651858. cd004403.pub2 [Crossref]

18. Anthonisen NR, Manfreda J, Warren CPW, et al. Antibiotic therapy in acute exacerbations of chronic obstructive pulmonary disease. Ann Intern Med 1987;106:196-204. [Crossref]

19. Jorgensen AF, Coolidge J, Pedersen PA, et al. Amoxicillin in treatment of acute uncomplicated exacerbations of chronic bronchitis. A double-blind, placebo-controlled multicentre study in general practice. Scand J Prim Health Care 1992;10:7-11. [Crossref]

20. Daniels JM, Snijders D, de Graaff CS, et al. Antibiotics in addition to systemic corticosteroids for acute exacerbations of COPD. Am J Respir Crit Care Med 2010;181:150-7. [Crossref]

21. Salwan AA, Spigt M, Laue J, et al. Predictors of treatment with antibiotics and systemic corticosteroids for acute exacerbations of asthma and chronic obstructive pulmonary disease in primary care. BMC Fam Pract 2015;24;16-40. [Crossref] 
22. Butler CC, Gillespie D, White P, et al. C-reactive protein testing to guide antibiotic prescribing for COPD exacerbations. N Engl J Medicine 2019;381:111-20. [Crossref]

23. Rohde GG, Koch A, Welte T, et al. Randomized double blind placebo-controlled study to demonstrate that antibiotics are not needed in moderate acute exacerbations of COPD--the ABACOPD study. BMC Pulm Med 2015;15:5. [Crossref]
24. Wilson R, Sethi S, Anzueto A, et al. Antibiotics for treatment and prevention of exacerbations of chronic obstructive pulmonary disease. J Infect 2013;67:497-515. [Crossref]

25. Phillips H, Van Hook CJ, Butler T, et al. A comparison of cefpodoxime proxetil and cefaclor in the treatment of acute exacerbation of COPD in adults. Chest 1993;104:1387-92. [Crossref]

26. Cscorbi I. Drug interactions principles, examples and clinical consequences. Dtsch Arztebl Int 2012;109:546-56. 